\title{
Estudo comparativo das medidas ecobiométricas pelos métodos de imersão e contato
}

\author{
Comparative study of the biometric measurements made by immersion \\ and contact techniques
}

\author{
Airton Leite Kronbauer ${ }^{1}$ \\ Fernando Leite Kronbauer ${ }^{2}$ \\ Cláudia Leite Kronbauer ${ }^{3}$
}

\begin{tabular}{|l|}
\hline RESUMO \\
\hline Objetivo: Comparar os achados clínicos ecobiométricos das medidas \\
dos métodos de imersão e contato. Métodos: O comprimento axial foi \\
medido em 120 ecobiometrias por onda “A” em 60 pacientes com catarata, \\
usando o método de imersão (não-contato) e de contato, em amostra \\
pareada. Resultados: A média do comprimento axial encontrada foi de \\
23,19 mm (DP 1,32) com o método de imersão e 22,93 mm (DP 1,32) com \\
o método de contato, usando a mesma sonda ultra-sônica. A diferença \\
de 0,255 mm (DP 0,3 ) foi estatisticamente significativa para o nível de $0,01$. \\
A diferença deve ser levada em conta quando se estima precisão no \\
cálculo de lente intra-ocular. A média do desvio padrão entre as repetidas \\
medidas no mesmo olho foi de 0,04 com o método de imersão e 0,19 com \\
o método de contato. A diferença de 0,15 foi estatisticamente significa- \\
tiva para o nível de 0,01 . A diferença deve ser levada em conta quando \\
se estima precisão na reprodutibilidade da técnica de exame. Conclusão: \\
Esses dados fornecem parâmetros que podem ser usados para monitorar \\
a prática clínica e para realizar outros estudos. \\
\hline
\end{tabular}

Descritores: Biometria; Ultra-sonografia; Catarata; Lentes intra-oculares; Implante de lentes intra-oculares; Facoemulsificação; Estudo comparativo; Reprodutibilidade de resultados

\section{INTRODUÇ̃̃O}

O exame ecobiométrico ocular se destina a realizar medidas oculares através do uso ultra-sonográfico da onda linear tipo "A"(1-4). Pode ser realizado através do contato direto da sonda com o globo ocular (método de contato/aplanação) ou pelo método de imersão (não-contato), através do uso de uma interface aquosa unindo a sonda ao olho ${ }^{(5)}$. Sua importância na prática clínica é o cálculo do valor dióptrico dos implantes de cristalino $\operatorname{artificial}^{(6)}$.

Com o crescente avanço da confiabilidade das cirurgias de implantes de lentes intra-oculares (LIOs) com finalidades refrativas secundárias (cirurgia de catarata) ou até mesmo primárias (cirurgias refrativas fácicas ou pseudofácicas), a precisão dos cálculos ecobiométricos é fundamental. Os fatores de interferência no resultado final são múltiplos com qualquer técnica e o erro pode ser cientificamente comprovado ${ }^{(1,7-9)}$, alcançando até 2,3 dioptrias em diferentes medidas no mesmo olho ${ }^{(10)}$ com propostas inclusive de técnicas de correção pós-implante ${ }^{(11-12)}$.

O objetivo deste trabalho é comparar as medidas ecobiométricas obtidas através dos métodos de contato e imersão e avaliar a reprodutibilidade das mesmas em subseqüentes medidas. 


\section{MÉTODOS}

Este estudo prospectivo selecionou consecutivamente 60 pacientes submetidos ao exame pré-operatório da catarata senil. Todos os pacientes realizaram ecobiometria através dos dois métodos, de imersão e de contato. Foram excluídos pacientes com cirurgia ocular prévia ou com outras doenças oculares e com doenças vítreas que causassem dificuldade na interpretação do gráfico de onda "A". Nos olhos selecionados eram aferidos 10 ecogramas em onda "A" pela técnica de contato e 10 ecogramas onda "A" pela técnica de contato.

Foram obtidas a média do comprimento axial do globo, a média do comprimento axial da câmara anterior, média do comprimento axial do cristalino, média do comprimento axial da cavidade vítrea e o desvio padrão entre os 10 ecogramas do comprimento axial do globo. As médias e os desvios realizados pelas duas diferentes técnicas foram pareadas no mesmo olho.

O aparelho usado foi o biômetro OcuScan ${ }^{\circledR} \mathrm{RxP}_{\text {da Alcon }}{ }^{\circledR}$ no padrão automático seriado de ondas tipo "A" com ganho aproximado de $65 \mathrm{~dB}$. Todas as medidas analisadas neste estudo foram realizadas em um único aparelho. Para controlar o viés de aferição do equipamento, foram comparados em estudo piloto prévio os resultados entre dois aparelhos idênticos da mesma geração de fabricação localizados em clínicas diferentes, com resultados similares nas medidas do comprimento axial e na diferença entre as técnicas.

A fórmula de SRK e SRK-T foi estabelecida como parâmetro de comparação com a constante A da LIO de 118,7.

Todos os exames foram realizados pelos dois primeiros autores que têm experiência de mais de 5 anos na realização de ecobiometria ultra-sônica de contato e cinco meses com a técnica de imersão. Não existe interesse comercial dos autores em nenhum tipo de equipamento oftalmológico.

Os métodos estatísticos usados foram os testes para variáveis quantitativas de regressão linear, teste t de regressão, teste t para amostras pareadas, coeficiente de relação de Pearson e análise dos dados pela curva de distribuição normal. $\mathrm{O}$ nível de significância estabelecido e pesquisado foi de 0,01 . Os gráficos apresentados foram calculados, geometricamente desenhados e superpostos por um programa estatístico baseado nos dados coletados.

\section{RESULTADOS}

O comprimento axial ocular médio dos 120 exames ultra-sônicos foi de 23,06 mm (Gráfico 1). A média dos valores do comprimento axial obtidos com a técnica de imersão foi de $23,19 \mathrm{~mm}$ e de 22,93 mm com a técnica de contato, sendo que curva de distribuição normal encontra-se no gráfico 2. Foi constatada correlação muito forte 0,974 pelo Coeficiente de Correlação de Pearson e a diferença de $0,255 \mathrm{~mm}$ entre os dois métodos mostrou significância de 0,01 pelo teste $t$ de amostras pareadas.

$\mathrm{O}$ desvio padrão entre as 10 medidas repetidas no mesmo olho foi usado como forma de comparação de reprodutibilidade do exame nos diferentes métodos. Portanto, quanto menor o desvio padrão entre as diversas medições, melhor a reprodutibilidade do exame e mais confiável o método. A média do desvio

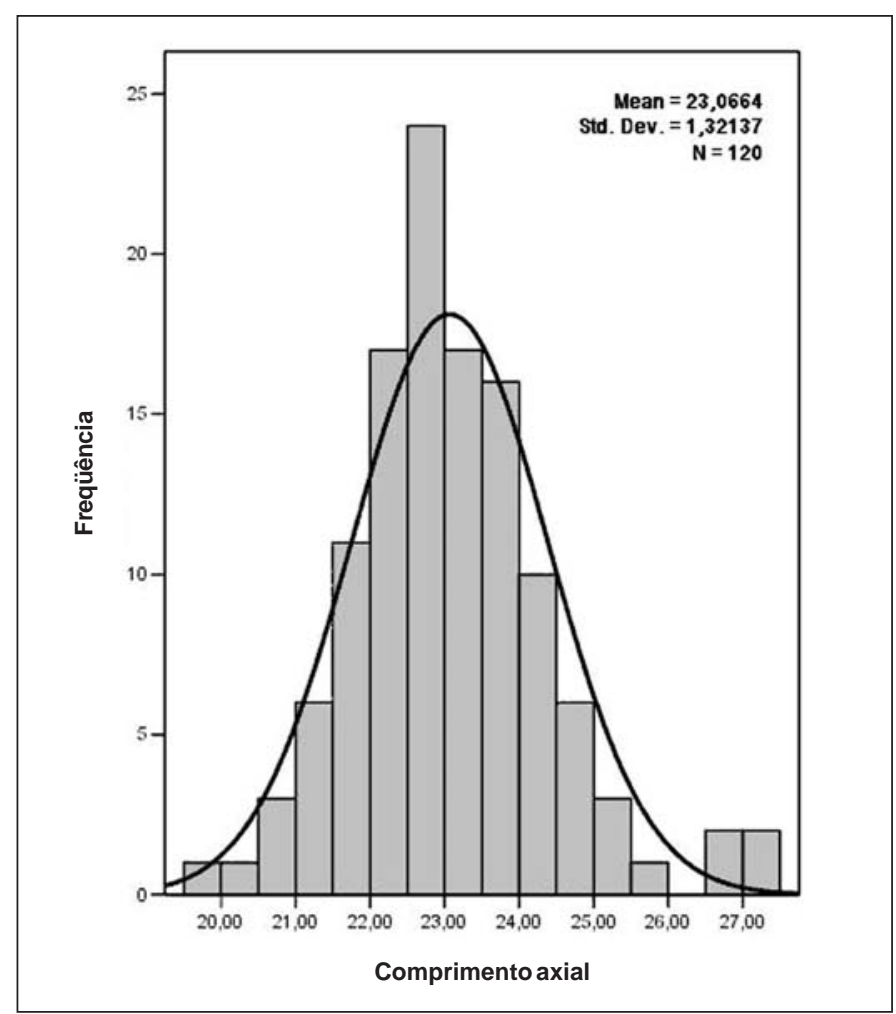

Gráfico 1 - Representação gráfica da distribuição de freqüência das medidas do comprimento axial na amostra estudada

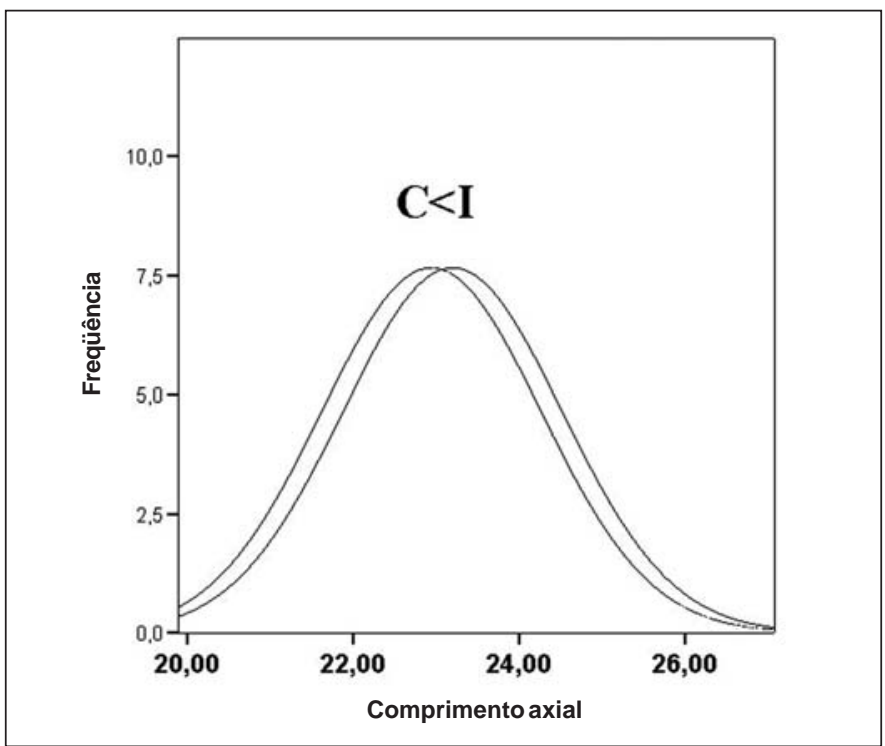

Gráfico 2 - Representação gráfica da comparação das curvas de distribuição normal do comprimento axial do globo ocular nos métodos de contato (curva da esquerda) e de imersão (curva da direita). Observar que a mediana do comprimento axial do método de contato $(C)$ é menor que o de imersão (I). * $p<0,01$ 
padrão de todas as medidas obtidas pelas biometrias foi de 0,11 (DP 0,09) (Gráfico 3). A média do desvio padrão no método de imersão foi de 0,04 com a curva de distribuição normal com dados desta amostra no gráfico 4. Enquanto que a média do desvio padrão no método de contato foi de 0,19 com a curva de distribuição normal com dados desta amostra no gráfico 4. Foi constatada correlação fraca de 0,284 e a diferença de 0,153 entre os dois métodos mostrou significância de 0,01 pelo teste $\mathrm{t}$ de amostras pareadas.

Quanto à subdivisão das medidas, os resultados da câmara anterior (média de 3,12 $\mathrm{mm}$ no contato e 3,23 $\mathrm{mm}$ na imersão) estão representados no gráfico 5 , os do cristalino (média de 4,41 mm no contato e 4,56 mm na imersão) no gráfico 6 e do vítreo (média de 15,392 mm no contato e 15,395 mm na imersão) no gráfico 7. Observou-se que a maior diferença entre os dois métodos encontra-se nas medidas da câmara anterior e cristalino e que a maior correlação e menor diferença encontra-se na medida do vítreo.

A diferença entre o comprimento axial ocular médio entre os dois métodos foi de $0,255 \mathrm{~mm}$ graficamente distribuída na amostra de regressão linear (Gráfico de dispersão 8). Essa média da diferença da medida do comprimento axial corresponde a uma diferença média no resultado do cálculo de implante de lente intra-ocular de 0,84 dioptrias pela fórmula SRK-T nesta amostra (Gráfico de dispersão 9). A diferença média de 0,84 dioptrias encontradas no valor da LIO corresponde a aproximadamente 0,6 dioptrias no grau do equiva-

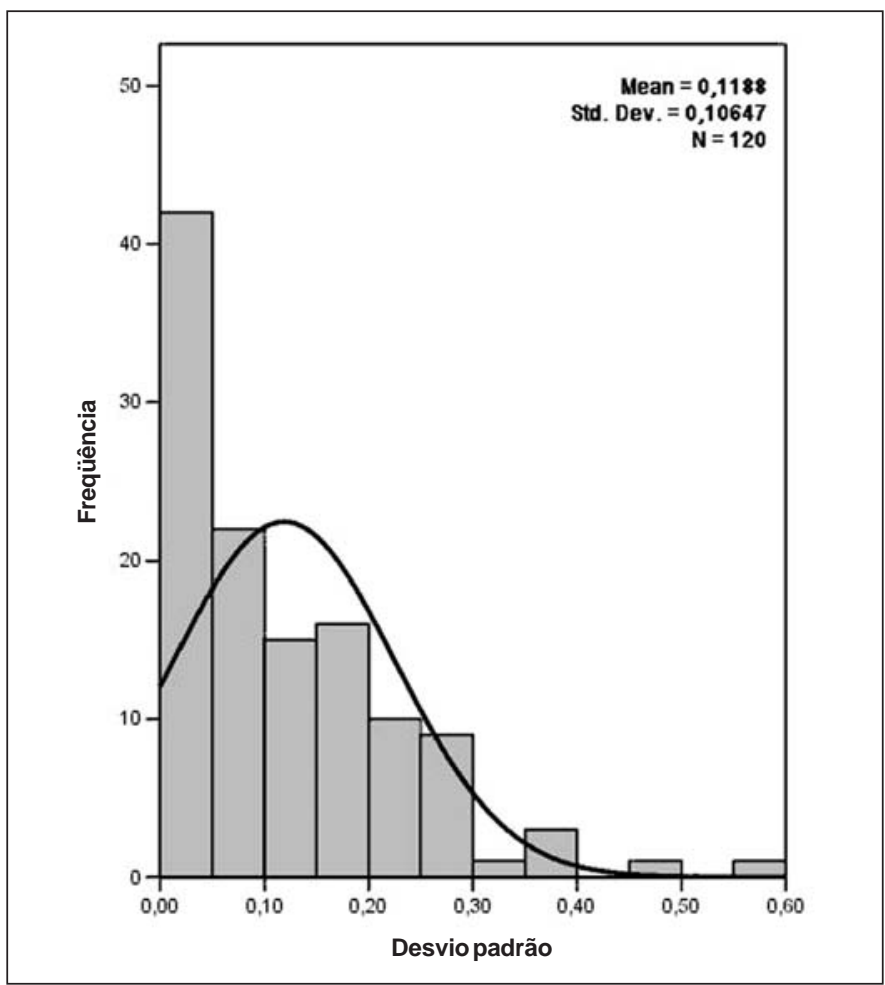

Gráfico 3 - Representação gráfica da distribuição de freqüência das medidas do desvio padrão de cada exame ecobiométrico lente esférico dos óculos, analisando os dados médios dessa amostra pela fórmula SRK-T para emetropia (Gráfico 10). Considerando o erro padrão médio $(0,128)$, a variação no grau da LIO estaria entre 0,71 a 0,97 dioptrias a menos, na técnica de imersão em relação à técnica de contato.

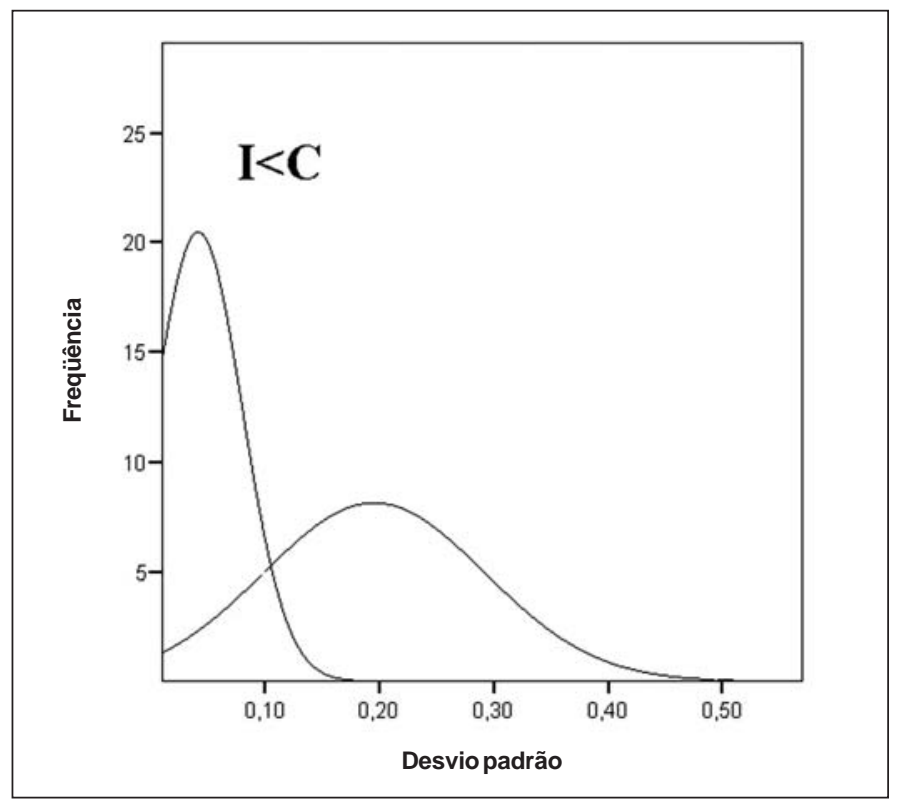

Gráfico 4 - Representação gráfica da comparação das curvas de distribuição normal do desvio padrão de cada exame ecobiométrico nos métodos de imersão (I - curva da esquerda) e de contato (C - curva da direita). Observar que nas repetidas medidas de cada exame ecobiométrico pelo método de imersão (I) existe menor amplitude de variação do que no método de contato (C). ${ }^{*} \mathrm{p}<0,01$

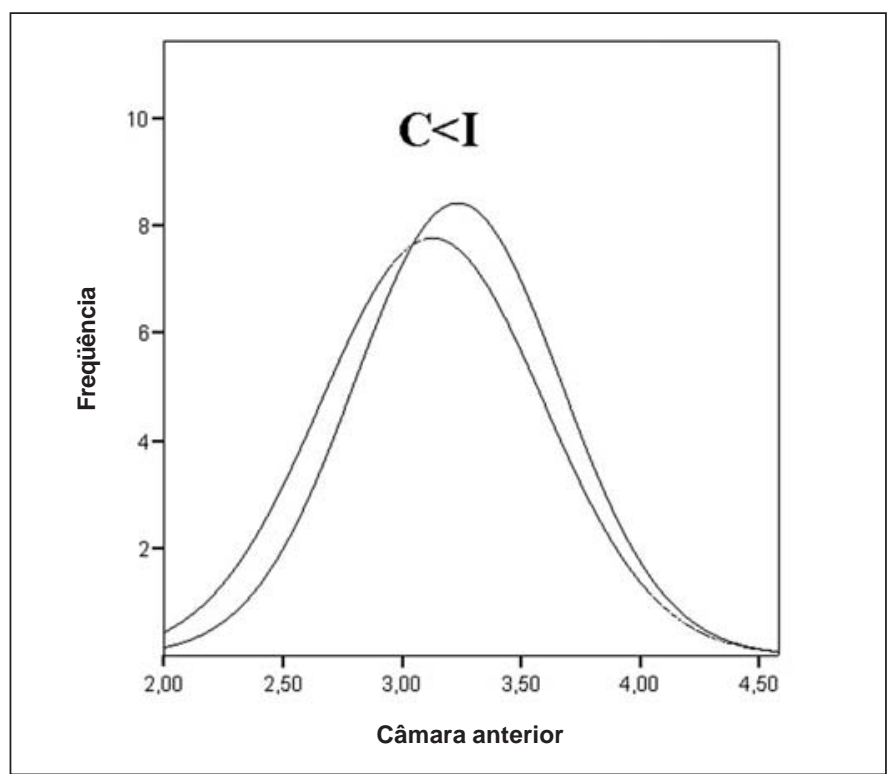

Gráfico 5 - Representação gráfica da comparação das curvas de distribuição normal do comprimento axial da câmara anterior nos métodos de contato ( $C$ - curva da esquerda) e de imersão (I - curva da direita). Observar que a mediana do método de contato (C) é menor que o de imersão (I). ${ }^{*} \mathrm{p}<0,01$ 
O índice de correlação de Pearson entre os métodos de imersão e contato foi de 0,974 com significância de 99\%. A regressão linear da correlação do valores do comprimento dos dois métodos está representada no gráfico de dispersão 8. Na avaliação do teste $\mathrm{t}$ para amostras pareadas, o valor de $\mathrm{t}$ da diferença entre os diâmetros foi de 6,523 para o intervalo de confiança de $99 \%$ (0,151-0,359). Portanto, a diferença de $0,255 \mathrm{~mm}$ na média dos diâmetros entre os dois métodos é estatisticamente significativa

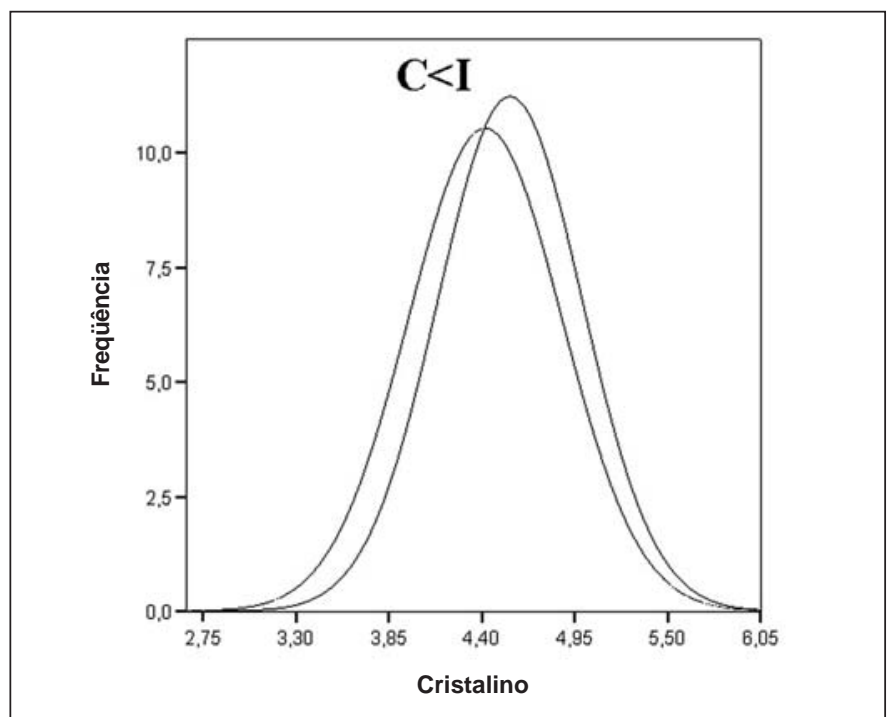

Gráfico 6 - Representação gráfica da comparação das curvas de distribuição normal do comprimento axial da cristalino nos métodos de contato (C - curva da esquerda) e de imersão (I - curva da direita). Observar que a mediana do método de contato (C) é menor que o de imersão (I). ${ }^{*} \mathrm{p}=0,01$

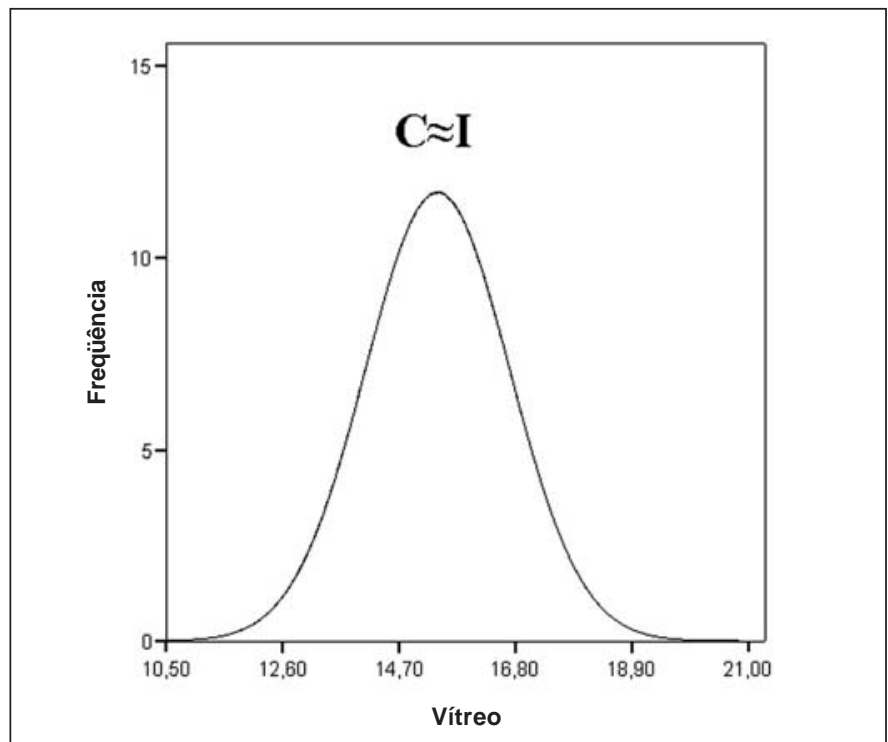

Gráfico 7 - Representação gráfica da comparação das curvas de distribuição normal do comprimento axial do vítreo nos métodos de contato (C - curva da esquerda) e de imersão (I - curva da direita). Observar que existe quase perfeita superposição gráfica entre os dois métodos. ${ }^{*} \mathrm{p}=0,94$ na amostra. Então, neste estudo o método de imersão mediu em média $0,255 \mathrm{~mm}$ a mais que o método de contato.

$\mathrm{Na}$ avaliação da reprodutibilidade e precisão, entre as diferentes medidas realizadas pelo mesmo método e a correlação da diferença do desvio padrão entre os dois métodos, encontramos no teste $t$ para amostras pareadas o valor de t de 12,73 para o intervalo de confiança de $99 \%(0,12-0,18)$. Portanto, a diferença de 0,153 na média do desvio padrão entre os dois métodos é estatisticamente significativa na amostra. Então, o método de

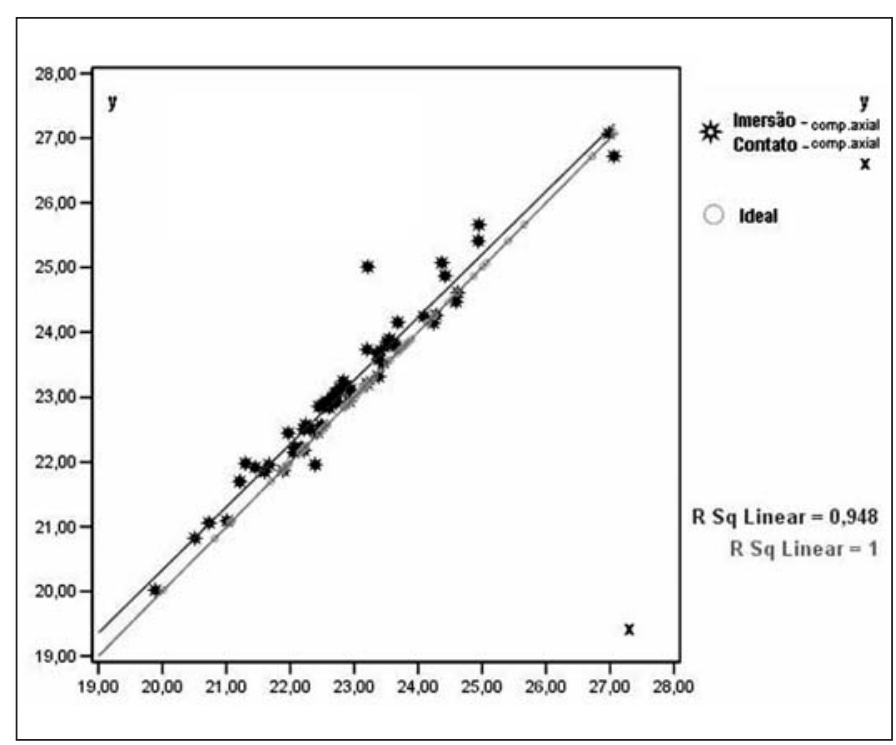

Gráfico 8 - Representação gráfica de dispersão das curvas de distribuição normal do comprimento axial do globo ocular nos métodos de contato $(x)$ e de imersão $(y)$. Observar a representação gráfica da regressão linear entre os dois métodos (linha superior) e a regressão linear ideal (linha inferior).

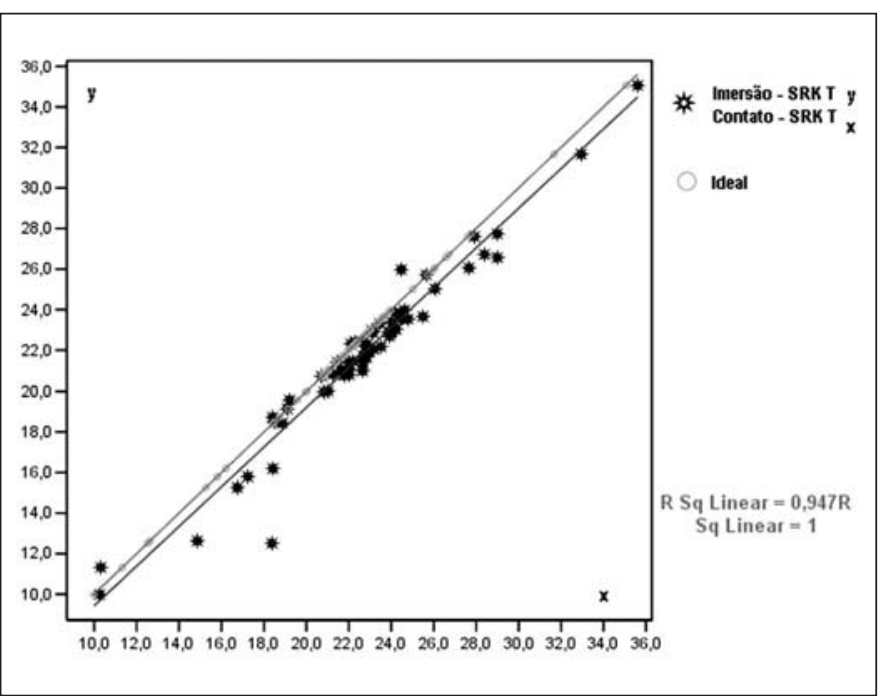

Gráfico 9 - Representação gráfica de dispersão das curvas de distribuição normal do resultado da fórmula SRK-T nos métodos de contato $(x)$ e de imersão $(y)$. Observar a representação gráfica da regressão linear entre os dois métodos (linha inferior) e a regressão linear ideal (linha superior). 


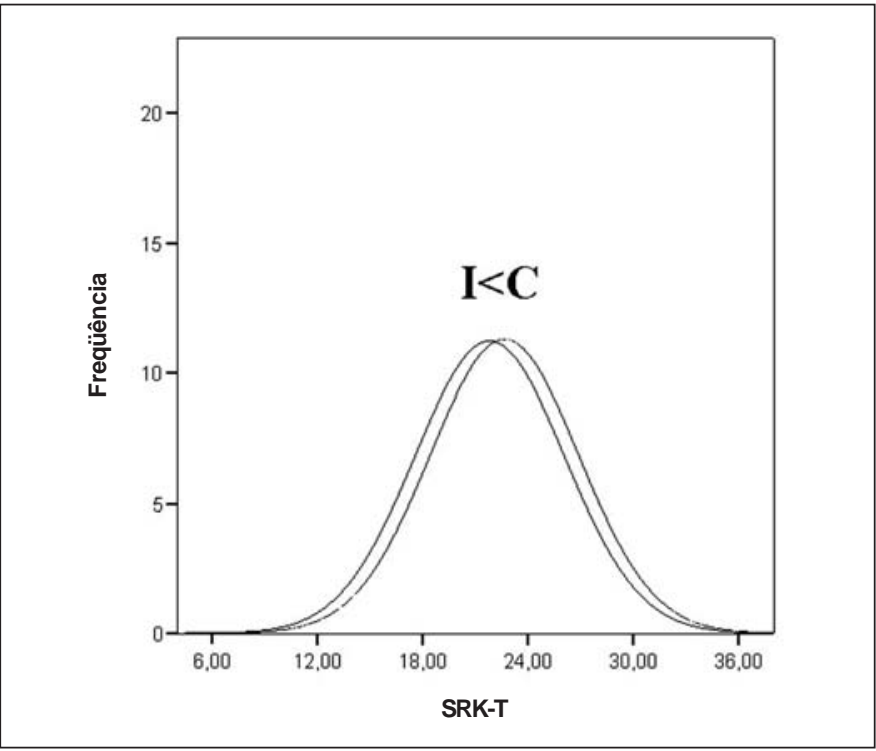

Gráfico 10 - Representação gráfica da comparação das curvas de distribuição normal do resultado da fórmula SRK-T nos métodos de imersão (curva da esquerda) e de contato (curva da direita). Observar que a mediana do método de imersão (I) é menor que o de contato (C). ${ }^{*} p<0,01$

imersão apresentou erro padrão médio de 0,04 menor e estatisticamente diferente que o erro padrão médio de 0,19 do método de contato. Houve, portanto, maior reprodutibilidade e precisão entre as várias medidas no método de imersão (Gráfico 4).

\section{DISCUSSÃO}

Na prática clínica e na literatura existem vantagens com as duas técnicas. As vantagens do método de contato são: praticidade e a rapidez; maior conforto, podendo o paciente estar deitado ou sentado; menor número de instrumentos; e menor chance de contaminação instrumental. As vantagens do método de imersão são: maior reprodutibilidade; não compressão da córnea; e alinhamento mais seguro em pacientes colaborativos ${ }^{(13-14)}$.

Alguns estudos atuais comparam a ecobiometria óptica com a ultra-sônica pela técnica de contato, apresentando maior comprimento axial na forma óptica ${ }^{(15)}$, sugerindo maior precisão no resultado final ${ }^{(16-18)}$. Haigis propôs correção da fórmula usada no cálculo final da ecobiometria óptica aproximando-se da fórmula teórica ${ }^{(6)}$.

Um dos trabalhos comparativos encontrados na literatura sobre ecobiometria de contato e de imersão não mostrou diferença significativa entre os métodos ${ }^{(19)}$. Contudo, outros autores encontraram resultados similares a esse estudo ${ }^{(20-21)}$ tanto no comprimento axial ocular quanto na reprodutibilidade, sendo sugerido $^{(21)}$ que a diferença pode ser variável dependendo do equipamento.

Os estudos das fórmulas de cálculo de lente intra-ocular são baseados principalmente no comprimento axial do globo ocular e poder dióptrico corneano. Os cálculos teóricos de implante foram corrigidos através de pesquisas clínicas com propostas de fórmulas com múltiplos fatores de correção ${ }^{(6,22-27)}$. Uma boa parte dessas pesquisas usa a regressão estatística dos dados clínicos encontrados para incorporar os fatores de correção nas fórmulas. É importante ressaltar que esses estudos foram baseados principalmente em dados ecobiométricos de contato que, conforme essa pesquisa, têm o valor do comprimento axial menor e reprodutibilidade menor que o método de imersão.

Avaliando os resultados sugere-se como padrão-ouro o estudo de imersão ao analisar reprodutibilidade; entretanto, torna-se indispensável que a interpretação das diversas fórmulas de cálculo de LIO existentes sejam corrigidas e avaliadas para esse tipo de método de realização do exame.

São muitas as causas de erros ecobiométricos no cálculo da LIO na prática clínica ${ }^{(1,6,8-10)}$. Esses erros estão nas fórmulas e suas constantes, na medida ceratométrica, na medida do comprimento axial ocular, na exata localização do implante no pósoperatório e nas particularidades individuais ${ }^{(1,28-29)}$. A medida ceratométrica e o poder refrativo da córnea estão sujeitos às interferências das aberrações corneanas, aos erros de aferição da medida automatizada ou manual ${ }^{(30-31)}$. A medida do diâmetro antero-posterior está sujeita a interferência dos métodos diagnósticos, dos índices, das velocidades, das densidades ecográficas e até dos diversos equipamentos ${ }^{(19-21)}$. As fórmulas são padronizadas e apenas parcialmente personalizadas, sendo suas constantes sujeitas a variações e erros práticos ${ }^{(31)}$. A previsão da localização pós-cirúrgica exata da distância córnea e lente intraocular pode também interferir no cálculo da lente ${ }^{(32-33)}$.

A experiência de vários anos dos examinadores com a técnica de contato e de alguns meses com a técnica de imersão podem favorecer ao viés de aferição e deve ser lembrado pelos leitores dessa pesquisa ao analisar seus resultados ${ }^{(8)}$. Sugerese para os cirurgiões que usam o método de contato com resultados previsíveis, ao trocar para o método de imersão, tenham o cuidado de observar a diferença de aproximadamente 0,84 dioptrias no cálculo final da LIO da sua fórmula de preferência. Sugerem-se novos estudos com amostragem maior para realizar normogramas e comparar fórmulas de cálculo de LIO diferentes para os dois métodos.

\section{CONCLUSÕES}

A padronização da interpretação dos dados ecobiométricos deve levar em conta o tipo de método empregado: contato ou imersão. O método de contato forneceu valores em média 0,255 mm a menos que o de imersão. A média do desvio padrão entre as repetidas medidas, usando como controle o mesmo olho, representa a reprodutibilidade do exame nos diferentes métodos. Portanto, quanto menor o desvio padrão entre as diversas medições, melhor a reprodutibilidade do exame e mais confiável o método. Concluímos que o método de imersão possui maior precisão e reprodutibilidade. Verificamos também que existem diferenças significativas entre as medidas dos dois métodos, necessitando; portanto, fórmulas distintas adaptadas para cálculos ecobiométricos de acordo com a técnica de exame empregada. 


\section{ABSTRACT}

Purpose: To compare clinical biometric findings between measurements of immersion technique and contact technique. Methods: Axial length was measured by A-scan in 120 medical examinations in 60 patients with cataract using a non-contact (immersion) and a contact technique in paired-samples by ultrasound. Results: The mean axial length was found to be $23.19 \mathrm{~mm}$ (SD 1.32) with the immersion technique and $22.93 \mathrm{~mm}$ (SD 1.32) with the contact technique, using the same transducer probe. The difference of $0.255 \mathrm{~mm}$ (SD 0.3) was significant at the 0.01 level. The difference should be taken into account when evaluating the accuracy of IOL calculation. The mean standard deviation between recurrent measures in same eye was found to be 0.04 with the immersion technique and 0.19 with the contact technique. The difference of 0.15 was significant at the 0.01 level. The difference should be taken into account when evaluating the accuracy of reproductivity of technique examination. Conclusion: These data provide benchmark information that can be used to monitor clinical practice and to perform others studies.

Keywords: Biometry; Ultrasonography; Cataract; Lenses, intraocular; Lens implantation, intraocular; Phacoemulsification; Comparative study; Reproducibility of results

\section{REFERÊNCIAS}

1. Corrêa ZMS, Kronbauer FL, Goldhardt R, Marcon IM, Bakowicz F. Precisão ecobiométrica da fórmula SRK/T na facoemulsificação. Arq Bras Oftalmol. 2001;64(3):233-7.

2. Bruckner NL. The role of A-scan ultrasonics in ophthalmology. Ann Ophthalmol. 1971;3(9):1014-5

3. Fledelius HC. Ultrasound in ophthalmology. Ultrasound Med Biol. 1997;23(3): 365-75.

4. Berges O, Puech M, Assouline M, Letenneur L, Gastellu-Etchegorry M. Bmode-guided vector-A-mode versus A-mode biometry to determine axial length and intraocular lens power. J Cataract Refract Surg. 1998;24(4):529-35.

5. Thijssen JM. The history of ultrasound techniques in ophthalmology. Ultrasound Med Biol. 1993;19(8):599-618. Comment in: Ultrasound Med Biol. 1994;20(8):827.

6. Haigis W. Pseudophakic correction factors for optical biometry. Graefes Arch Clin Exp Ophthalmol 2001;239(8):589-98.

7. Murphy C, Tuft SJ, Minassian DC. Refractive error and visual outcome after cataract extraction. J Cataract Refract Surg. 2002;28(1):62-6.

8. Findl O, Kriechbaum K, Sacu S, Kiss B, Polak K, Nepp J, et al. Influence of operator experience on the performance of ultrasound biometry compared to optical biometry before cataract surgery. J Cataract Refract Surg. 2003;29(10):1950-5.

9. Pierro L, Modorati G, Brancato R. Clinical variability in keratometry, ultrasound biometry measurements, and emmetropic intraocular lens power calculation. J Cataract Refract Surg. 1991;17(1):91-4.

10. Norrby S. Multicenter biometry study of 1 pair of eyes. J Cataract Refract Surg. 2001;27(10):1656-61.

11. Schwartz DM, Sandstedt CA, Chang SH, Kornfield JA, Grubbs RH. Light- adjustable lens: development of in vitro nomograms. Trans Am Ophthalmol Soc. 2004;102:67-72; discussion 72-4

12. Mamalis N. Complications of foldable intraocular lenses requiring explantation or secondary intervention-2001 survey update J Cataract Refract Surg. 2002;28(12):2193-201.

13. Tehrani M, Krummenauer F, Blom E, Dick HB. Evaluation of the practicality of optical biometry and applanation ultrasound in 253 eyes. J Cataract Refract Surg. 2003;29(4):741-6.

14. Velazquez-Estades LJ, Wanger A, Kellaway J, Hardten DR, Prager TC. Microbial contamination of immersion biometry ultrasound equipment. Ophthalmology. 2005;112(5):e13-8.

15. Tehrani M, Krummenauer F, Kumar R, Dick HB. Comparison of biometric measurements using partial coherence interferometry and applanation ultrasound. J Cataract Refract Surg. 2003;29(4):747-52.

16. Rajan MS, Keilhorn I, Bell JA. Partial coherence laser interferometry vs conventional ultrasound biometry in intraocular lens power calculations. Eye. 2002;16(5):552-6.

17. Drexler W, Findl O, Menapace R, Rainer G, Vass C, Hitzenberger CK, et al. Partial coherence interferometry: a novel approach to biometry in cataract surgery. Am J Ophthalmol. 1998;126(4):524-34.

18. Connors R 3rd, Boseman P 3rd, Olson RJ. Accuracy and reproducibility of biometry using partial coherence interferometry. J Cataract Refract Surg. 2002; 28(2):235-8.

19. Hennessy MP, Franzco, Chan DG. Contact versus immersion biometry of axial length before cataract surgery. J Cataract Refract Surg. 2003;29(11):2195-8.

20. Schelenz J, Kammann J. Comparison of contact and immersion techniques for axial length measurement and implant power calculation. J Cataract Refract Surg. 1989;15(4):425-8.

21. Olsen T, Nielsen PJ. Immersion versus contact technique in the measurement of axial length by ultrasound. Acta Ophthalmol (Copenh). 1989;67(1):101-2.

22. Sanders DR, Retzlaff JA, Kraff MC, Gimbel HV, Raanan MG. Comparison of the SRK/T formula and other theoretical and regression formulas. J Cataract Refract Surg. 1990;16(3):341-6.

23. Retzlaff JA, Sanders DR, Kraff MC. Development of the SRK/T intraocular lens implant power calculation formula. J Cataract Refract Surg. 1990;16(3): 333-40. Erratum in: J Cataract Refract Surg 1990;16(4):528. Erratum in: J Cataract Refract Surg 19901;16(4):528. Comment in: J Cataract Refract Surg. 1993;19(3):442-6.

24. Sanders DR, Retzlaff J, Kraff MC. Comparison of the SRK II formula and other second generation formulas. J Cataract Refract Surg. 1988;14(2):136-41.

25. Sanders D, Retzlaff J, Kraff M, Kratz R, Gills J, Levine R, et al. Comparison of the accuracy of the Binkhorst, Colenbrander, and SRK implant power prediction formulas. J Am Intraocul Implant Soc. 1981;7(4):337-40.

26. Sanders DR, Retzlaff J, Kraff MC. Comparison of empirically derived and theoretical aphakic refraction formulas. Arch Ophthalmol. 1983;101(6):965-7.

27. Hoffer KJ. Biometry of 7,500 cataractous eyes. Am J Ophthalmol. 1980;90(3): 360-8.

28. Olsen T, Corydon L, Gimbel H. Intraocular lens power calculation with an improved anterior chamber depth prediction algorithm. J Cataract Refract Surg. 1995;21(3):313-9.

29. Elder MJ. Predicting the refractive outcome after cataract surgery: the comparison of different IOLs and SRK-II v SRK-T. Br J Ophthalmol. 2002;86(6):620-2.

30. Pierro L, Modorati G, Brancato R. Clinical variability in keratometry, ultrasound biometry measurements, and emmetropic intraocular-lens power calculation. J Cataract Refract Surg. 1991;17(1):91-4.

31. Oliveira FD, Muccioli C, Lopes YC, Soriano ES, BelfortJr R. Biometrias óptica e ultra-sônica: Comparação dos métodos usados para o cálculo da lente intra-ocular acomodativa. Arq Bras Oftalmol. 2004;67(6):887-91.

32. Minassian DC, Rosen P, Dart JK, Reidy A, Desai P, Sidhu M, et al Extracapsular cataract extraction compared with small incision surgery by phacoemulsification: a randomised trial. Br J Ophthalmol. 2001;85(7):822-9. Erratum in: Br J Ophthalmol 2001;85(12):1498. Erratum in: Br J Ophthalmol 2001;85(12):1498. Comment in: Br J Ophthalmol. 2001;85(7):765-6.

33. Olsen T. Sources of error in intraocular-lens power calculation. J Cataract Refract Surg. 1992;18(2):125-9. 